\title{
Human Sulfatase 2 inhibits in vivo tumor growth of MDA-MB-231 human breast cancer xenografts
}

\author{
Sarah M Peterson ${ }^{1}$, Andrea Iskenderian², Lynette Cook², Alla Romashko², Kristen Tobin', Michael Jones', \\ Angela Norton², Alicia Gómez-Yafal ${ }^{2}$, Michael W Heartlein², Michael F Concino², Lucy Liaw', Paolo GV Martini2 ${ }^{2}$
}

\begin{abstract}
Background: Extracellular human sulfatases modulate growth factor signaling by alteration of the heparin/heparan sulfate proteoglycan (HSPG) 6-O-sulfation state. HSPGs bind to numerous growth factor ligands including fibroblast growth factors (FGF), epidermal growth factors (EGF), and vascular endothelial growth factors (VEGF), and are critically important in the context of cancer cell growth, invasion, and metastasis. We hypothesized that sulfatase activity in the tumor microenvironment would regulate tumor growth in vivo.
\end{abstract}

Methods: We established a model of stable expression of sulfatases in the human breast cancer cell line MDA-MB231 and purified recombinant human Sulfatase 2 (rhSulf2) for exogenous administration. In vitro studies were performed to measure effects on breast cancer cell invasion and proliferation, and groups were statistically compared using Student's t-test. The effects of hSulf2 on tumor progression were tested using in vivo xenografts with two methods. First, MDA-MB-231 cells stably expressing hSulf1, hSulf2, or both hSulf1/hSulf2 were grown as xenografts and the resulting tumor growth and vascularization was compared to controls. Secondly, wild type MDA-MB-231 xenografts were treated by short-term intratumoral injection with rhSulf2 or vehicle during tumor growth. Ultrasound analysis was also used to complement caliper measurement to monitor tumor growth. In vivo studies were statistically analyzed using Student's t test.

Results: In vitro, stable expression of hSulf2 or administration of rhSulf2 in breast cancer cells decreased cell proliferation and invasion, corresponding to an inhibition of ERK activation. Stable expression of the sulfatases in xenografts significantly suppressed tumor growth, with complete regression of tumors expressing both hSulf1 and hSulf2 and significantly smaller tumor volumes in groups expressing hSulf1 or hSulf2 compared to control xenografts. Despite significant suppression of tumor volume, sulfatases did not affect vascular density within the tumors. By contrast, transient exogenous treatment of MDA-MB-231 xenografts with rhSulf2 was not sufficient to inhibit or reverse tumor growth.

Conclusion: These data indicate thatin vivo progression of human breast cancer xenografts can be inhibited with sulfatase expression, and therapeutic effect requires constant delivery at the tumor site. Our results support a direct effect of sulfatases on tumor growth or invasion, rather than an effect in the stromal compartment.

\section{Background}

Breast cancer is the leading cause of invasive cancer in women. The lifetime risk of breast cancer in women is estimated at 1 in 8 (13\% of women) [1]. Factors that decrease cancer cell invasion and tumor growth have the potential for translation into novel therapeutic approaches for lessening breast cancer morbidity and mortality.

\footnotetext{
* Correspondence: pmartini@shire.com

${ }^{2}$ Shire Human Genetic Therapies Inc., 700 Main Street, Cambridge, MA 02139, USA

Full list of author information is available at the end of the article
}

Extracellular sulfatases appear to have a significant role in cancer biology. Heparan sulfate-like glycosaminoglycans, along with structural proteins, are important regulators at the cell surface-extracellular matrix (ECM) interface [2,3]. Human Sulfatase 1 (hSulf1) and Sulfatase 2 (hSulf2) are heparan sulfate 6-O-endosulfatases, a family of secreted enzymes that are either localized on the cell surface or released into the ECM. HSulf1 and hSulf2 cleave 6-Osulfate moieties of heparan sulfate, thereby affecting composition and function of the glycosaminoglycans. Because glycosaminoglycans regulate cytokine signaling, heparan

\section{Ciomed Central}


sulfate sulfation patterns and pattern-dependent cell signaling events rely on activity of sulfatase enzymes. HSPGs are key components of the ECM that are involved in tumor progression by regulation of growth factor signaling pathways. Most of the molecular events associated with tumor growth, neovascularization, and metastases are influenced by interactions between cells and their ECM. There is mounting evidence for the role of two secreted human extracellular sulfatases, hSulf1 and hSulf2, in modulating the growth factor signaling pathways needed for tumor angiogenesis and progression [4]. However, much of the data that exists on the role of hSulf1 and hSulf 2 is context dependent to distinct cancer types and cellular environment [5-8].

HSulf1 and hSulf2 have overlapping yet distinct roles both in development and cancer progression. Mouse knockout models of Sulfatase 1 or Sulfatase 2 display a normal phenotype by nearly all criteria examined [9]. Combined knockout of Sulfatase 1 and 2 in mice, however, leads to $\sim 50 \%$ neonatal lethality and $\sim 80 \%$ postnatal lethality with prominent skeletal and renal developmental defects [9]. Cancer models also display evidence of context-specific sulfatase activity. HSulf1 expression is decreased in $82 \%$ of hepatocellular carcinoma (HCC) cell lines, leading to increased HSPG sulfation, enhanced FGF-mediated and hepatocyte growth factor (HGF)-mediated signaling, and increased cell growth [10]. Forced expression of hSulf1 decreases sulfation, inhibits growth factor signaling, and sensitizes HCC cells to chemotherapeutic apoptosis [10]. While previous study of overexpression of hSulf1 in the estrogen receptor negative MDA-MB-468 cell line has been shown to decrease tumor burden in athymic nude mice in vivo, few data are available regarding the therapeutic role of forced expression of hSulf2 on in vivo tumor burden.

We hypothesized that hSulf2 might have an effect in inhibiting cancer cell growth and invasion in vitro and in vivo. In order to further understand the role of hSulf2 in breast cancer, we chose the human breast cancer cell line MDA-MB-231, a well characterized and established model for human breast cancer growth. Although reports in the literature describe variable levels of sulfatase expression in MDA-MB-231 cells $[6,11]$, we did not detect endogenous expression of hSulf1 or hSulf2 in our MDA-MB-231 population, using RT-PCR. We therefore chose the MDA-MB-231 cell line for gain-of-function studies by creating transfected pools that stably express human sulfatases. Given that hSulf1 has been established as an inhibitor of tumorigenesis [8], we used overexpression of hSulf1 as a positive control and also tested hSulf1 in conjunction with hSulf2. For delivery of rhSulf2, intratumoral injection was chosen as the initial approach for two reasons. The first was based on in vivo data about the anticipated effect of hSulf 2 on the stromal component. The second was based on attempt to avoid first pass metabolism through the liver and deliver maximal protein to the tumor site.

In vitro studies provide evidence that expression or delivery of hSulf2 decreases proliferation and tumor cell invasion through the ECM. In vivo studies show that hSulf2 expression is effective in suppressing xenograft growth. In our system, this effect appears to be independent of an angiogenic effect, because tumor growth was significantly suppressed without dramatic changes in the angiogenic response to the xenograft. Further studies are needed to better characterize and validate the role of hSulf2 in inhibiting tumor growth and progression in vivo.

\section{Methods \\ Materials}

FGF2 and HB-EGF-2 were obtained from Sigma (St. Louis, MO). EGFR inhibitor PD153035 was obtained from EMD Biosciences (San Diego, CA). Total ERK and phospho-ERK antibodies were purchased from R\&D Systems (Minneapolis, MN). Growth factor reduced Matrigel was obtained from BD Biosciences (San Jose, CA). CD31 (PECAM) antibody was obtained from BD Pharmingen. Ki-67 antibody was obtained from Ventana Corporation (Tucson, AZ).

\section{Recombinant protein production}

An expression vector with full-length human Sulfatase 2 cDNA was transfected into HT1080 cells by electroporation. HT1080 cells were grown and maintained in a serum-free DMEM/F-12 based custom media (Invitrogen, Carlsbad, CA) at $37^{\circ} \mathrm{C}$ in a $5 \% \mathrm{CO}_{2}$ incubator. Transfection with a neomycin resistance cassette allowed for the selection of stably transfected clones. Clones were expanded and cell supernatants examined for expressed hSulf2 protein. Conditioned medium was processed by copper binding followed by gel filtration. Selected gel filtration fractions were pooled and dialyzed against $20 \mathrm{mM}$ sodium phosphate, $0.5 \mathrm{M} \mathrm{NaCl}, 10 \%$ glycerol, $0.5 \mathrm{mg} / \mathrm{ml}$ Pefabloc at $\mathrm{pH}=7.5$. RhSulf2 was visualized by Coomassie staining (GelCode Blue Stain Reagent, Pierce, Rockford, IL) of $8-16 \%$ tris-glycine SDS-PAGE gels (Invitrogen, Carlsbad, CA). Glycerol and Pefabloc were removed by dialysis into a final buffer of $500 \mathrm{mM} \mathrm{NaCl}, 20 \mathrm{mM} \mathrm{NaPO} 4 \mathrm{pH}=7$.

\section{Cell Culture}

MDA-MB-231 (HTB-26) and MDA-MB-435 S (HTB129) cell lines were obtained from ATCC. MDA-MB-231 cells stably expressing control vector, human Sulfatase 1 (hSulf1), human Sulfatase 2 (hSulf2), or a combination of 
human Sulfatase1 and 2 (hSulf1/hSulf2) were established. Specific clones of transfected hSulf1 and hSulf2 cells were selected based on the strength of respective hSulf protein and phenotypic validation in vitro of reduced cell proliferation and migration. MDA-MB-231 and MDAMB-435 S cells for in vitro experiments were cultured in minimal essential media (MEM) supplemented with $10 \%$ FBS, $2 \mathrm{mM} \mathrm{L}$-glutamine and $0.01 \mathrm{mg} / \mathrm{ml}$ insulin at $37^{\circ} \mathrm{C}$, $5 \% \mathrm{CO}_{2}$. For the invasion assay, cells were cultured in MEM supplemented with 5\% charcoal-stripped FBS, 2 $\mathrm{mM}$ L-Glutamine and $0.01 \mathrm{mg} / \mathrm{ml}$ insulin 24 hours prior to assay. On the day of the assay, cells were suspended in migration media (serum-free basal media) and placed in the top well of invasion chambers (Chemicon ECM554). Chemoattractant (10\% FBS) was placed in the lower chamber in migration media. Cells were allowed to invade for 24 hours at $37^{\circ} \mathrm{C}$. Cells were harvested and invasion rate was determined according to manufacturer's protocol. For MTT assays, cells were seeded at 40,000 cells $/ \mathrm{cm}^{2}$ into 48 -well plates in complete growth medium. After $24 \mathrm{~h}$, cells were rinsed and treated with either rhSulf2 formulation buffer or with varying concentrations of rhSulf2. On days 1, 2 and 3, $5 \mathrm{mg} / \mathrm{ml} \mathrm{MTT}$ was added to the cells for 4 hours at $37^{\circ} \mathrm{C}$. After the incubation the media was aspirated, DMSO was added and the OD at $570 \mathrm{~nm}$ was measured. Cell count and viability was assessed using a Cell sorter (Cedex, Innovatis-Roche, Germany). For tumor xenograft implantation, MDA-MB231 cell populations expressing human sulfatases were cultured in MEM supplemented with $10 \%$ FBS, 1\% nonessential amino acids, and $0.5 \%$ gentamicin.

\section{RT-PCR}

Primers used for hSulf1 were S1fw $5^{\prime}$ ACGGGGGAGCTGGAGAATACTTAC 3'/S1rev 5' GCCACT TCTGCCCCGGTTGTTCAC 3', for hSulf2 there were 2 sets of primers S2fw 5' CCGCCCAG CCCCGAAACC 3'/S2rev 5' CTCCCGCAACAGCCACACCTT 3' as well as S2fw 5' CTCCGTTTTCCT TTGTGAGC 3'/S2rev 5' GAATTTGCAACTGGCTT CCT $3^{\prime}$ and for $\beta$-actin $5^{\prime}$ AGAAAATCTGGCACCACACC $3^{\prime} / 5^{\prime}$ CTCCTTAATGT CACGCACGA 3' was used. One Step RT-PCR kit (Invitrogen, Carlsbad, CA) was used according to manufacturer's instruction for sulfatase determination in cells.

\section{Stable transfectant tumor xenograft production}

All animal studies were performed in accordance with established protocols approved by the Maine Medical Center Institutional Animal Care and Use Committee. Three groups of stably transfected MDA-MB-231 cells were prepared for in vivo tumor xenograft growth in comparison with control vector-transfected cells: cells expressing both hSulf1 and hSulf2, only hSulf1, or only hSulf2. Thirty-two litter-matched female $\mathrm{NCr}$ homozygous $n u / n u$ mice (Taconic) at nine weeks of age were chosen as xenograft hosts. These were randomized by cage into four groups of eight mice each. The first group was injected with the control vector-transfected cells, the second group was injected with cells expressing both hSulf1 and hSulf2, the third group was injected with cells expressing only hSulf1, and the fourth group was injected with cells expressing only hSulf2. Injections were placed subcutaneously into the left flank and contained 5 million MDA-MB-231 cells suspended in $200 \mu \mathrm{l}$ of PBS. Measurements were obtained by caliper length and width measurements at 2-3 day intervals for the duration of the experiment. Tumor volume was calculated from the pi-based ellipsoid volume formula $\pi / 6$ "length*width*height [12], assuming ellipsoid shape with equal width and height. The average value and standard deviation are based on calculated tumor volumes from the eight mice in each group. Tumor xenografts recovered from mice were fixed in $4 \%$ paraformaldehyde and embedded in paraffin. Tissue sections were stained with Masson's trichrome staining for visualization of collagen, CD31 (PECAM) staining for visualization of tumor vasculature, or $\mathrm{Ki}-67$ staining for mitotic index. Average mitotic index quantification was obtained by counting 5 high-power fields per tumor using ImageJ software [13].

Tumor xenograft production for exogenous therapy with purified recombinant human Sulfatase 2 (rhSulf2)

For production of tumor xenografts for the exogenous treatment arm of the study, $200 \mu \mathrm{l}$ of a 1:1 Matrigel:buffer suspension containing 8.5 million MDA-MB-231 cells was subcutaneously injected into the left flank of 32 female litter-matched nude mice. Four additional female litter-matched nude mice were injected with 200 $\mu \mathrm{l}$ of the Matrigel:buffer suspension only, and four remaining female litter-matched nude mice were left un-manipulated as experimental controls. Following a 48 hour window to allow establishment of tumor xenografts in mice injected with tumor cells, intratumoral injections of rhSulf2 were administered to the 16 mice randomized to the treatment group. Each injection contained $0.1 \mathrm{mg}$ rhSulf 2 based on an estimated $5 \mathrm{mg} / \mathrm{kg}$ dose in an average $20 \mathrm{~g}$ nude mouse. An equal volume of control buffer vehicle was administered intratumorally to the 16 mice in the treatment control group.

\section{High resolution ultrasound imaging of tumor xenografts}

Mice were anesthetized by inhaled isoflurane prior to ultrasound scanning with a VisualSonics Vevo 770 highresolution imaging system. Tumors were initially scanned free hand to establish approximate tumor size and morphology. Images for tumor reconstruction were acquired by $3 \mathrm{D}$ motor stage in alignment with the long 
axis of the tumor. After scanning, images were processed on a high-definition monitor for obtaining volumetric measurements. Successive tracings of tumor contour were taken which enabled the computer software to generate a 3D image and volume for each tumor. For comparison to external caliper measurements, $x$ axis (length) was assigned to the longest visible dimension of the tumor. From there, y axis (width) was assigned to 90 perpendicular axis, and $\mathrm{Z}$ axis (height) assigned to tumor depth. The pi-based ellipsoid equation $\pi / 6 *$ length"width*height has previously been validated as the best equation for estimating subcutaneous tumor size in athymic nude mice [12]. However, tracking of tumor size by estimation of standard caliper measurements in vivo involves the assumption of width equaling depth, given that only two dimensions can be measured in vivo. Here we report methodology for making these measurements more precise by non-invasively capturing depth component through high resolution in vivo ultrasound imaging.

\section{Statistical Analysis}

Statistical analyses were performed using Student's t test, with a significant difference determined as $\mathrm{p}<0.05$. Where appropriate, data are represented as means \pm SD.

\section{Results}

\section{HSulf2 expression in MDA-MB-231 cells}

Most of the recent data have been focused on the effect of hSulf1 in different tumor cell lines. HSulf1 overexpression in cells has been reported to inhibit tumor formation in vitro and in vivo. We were interested in understanding the role of the related protein hSulf2 in breast cancer. While MDA-MB-435 S cells expressing Sulfatase 2 can represent a valid model to study the effect of endogenous Sulfatase 2 in breast cancer, we have chosen MDA-MB-231 cells as a model for our study because they have undetectable levels of endogenously produced human Sulfatase 1 or Sulfatase 2 as analyzed by RT-PCR (Fig. 1A). MDA-MB-435 S cells in comparison have detectable endogenous expression of Sulfatase 2 as shown in Fig. 1A. In addition, MDA-MB231 is an estrogen-independent cell line that does not require exogenously added estrogen for xenograft growth. This was an advantage in our study to focus specifically on changes in sulfatases, because estrogen has diverse effects on tumor phenotype and angiogenesis $[14,15]$. Thus, MDA-MB-231 cells were used for gainof-function studies to understand their roles in breast cancer cell phenotype. We generated MDA-MB-231 cells that stably express human Sulfatase 2 or Sulfatase 1 , or a combination of both sulfatases. HSulf1 stably transfected MDA-MB-231 cells were used as a positive control based on previous publication on the effect of expression of hSulf1 in breast cancer cells [8]. Following transfection with sulfatase constructs, expression was confirmed by RT-PCR with specific primers (Fig. 1B). In addition, we were interested in understanding the effect of recombinant hSulf2 as a possible therapeutic for breast cancer. We have expressed and purified recombinant hSulf2 (rhSulf2) in the HT1080 human cell line. Coomassie staining shows a prominent band at about $120 \mathrm{KDa}$, corresponding to rhSulf2 loaded in both lanes (Fig. 1C). After purification, rhSulf2 was tested for activity based on its ability to convert 4-methylumbelliferyl sulfate (4-MUS) into fluorescent 4-methylumbelliferone as previously described [16]. The activity of hSulf2 was relatively stable over the course of 2 days if diluted in mouse serum, which had a protective effect on rhSulf2, and extended its biological activity over time compared to rhSulf 2 stored in cell medium at $37^{\circ} \mathrm{C}$ (Fig. 1D). These results suggest that rhSulf 2 could potentially be used for in vivo studies due to its long lasting activity in serum.

\section{Modulation of MDA-MB-231 cell invasion and proliferation by hSulf2}

The effect of sulfatases in inhibiting cell invasion has been investigated by over-expression of hSulf1 in different cancer cells $[4,8]$. But, to date, recombinant human Sulfatase 2 effect on breast cancer cells has not been tested in vitro or in vivo. We treated MDA-MB-231 cells with different concentrations of rhSulf2 and we observed a dose-dependent decrease in invasion through an ECM barrier with the highest effect at $125 \mathrm{nM}$ or $250 \mathrm{nM}$ rhSulf2 compared to buffer control (Fig. 2A). As a positive control we tested MDA-MB-231 cells that were stably transfected with either hSulf1 or hSulf2. HSulf1 was shown by Narita et al. [8] to decrease invasion of MDA-MB-468 breast cancer cells. HSulf1 and hSulf2 stably transfected MDA-MB-231 cells confirmed previous data from MDA-MB-468 cells and our results obtained by treating the cells with rhSulf2 also showed inhibition of cell invasion. It is interesting to note that when we looked at the invasion rate of MDA-MB-435 $S$ cells, expressing endogenous Sulfatase 2, we have observed a lower degree of invasion compared to MDAMB-231 cells. The lower invasion rate of MDA-MB-435 $\mathrm{S}$ was further suppressed by adding $250 \mathrm{nM}$ rhSuf2, suggesting that hSulf 2 may play a role in regulating metastases in breast cancer. Furthermore, rhSulf2 inhibited invasion at a comparable rate to the known EGFRdependent kinase inhibitor PD 153035 [17] (Fig. 2B). Given the role of growth factors in promoting cell proliferation and knowing that sulfatases might influence the interaction of the growth factor receptors with signaling molecules, we tested the ability of hSulf 2 to inhibit cell proliferation in vitro. We measured cellular 


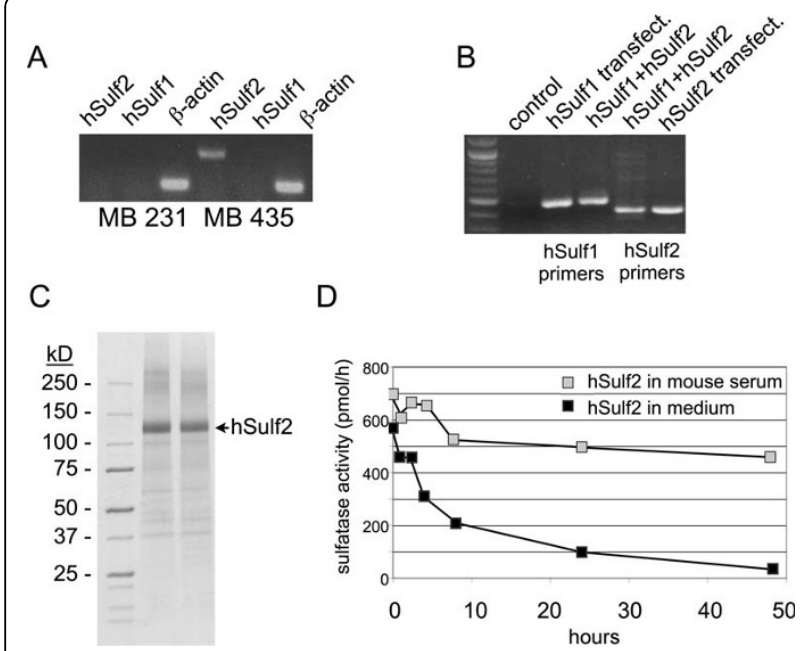

Figure 1 Sulfatase expression in a human breast cancer model and analysis of recombinant human Sulfatase 2. A) Human breast cancer cell lines MDA-MB-231 (MB 231) and MDA-MB-435 S (MB 435) were tested for expression of Sulfatase1 (hSulf1), Sulfatase2 (hSulf2), or $\beta$-actin by RT-PCR. B) RT-PCR was used to detect hSulf1, hSulf2 or both sulfatase transcripts in MDA-MB-231 after stable transfection with human sulfatase-containing plasmids. C) Coomassie staining of SDS-PAGE gel shows a prominent band at $120 \mathrm{KDa}$ corresponding to purified rhSulf2 loaded in both lanes. D) Purified hSulf2 protein was incubated at $250 \mathrm{nM}$ in medium or mouse serum, and activity assayed over time.

metabolic activity (Fig. 2C) of MDA-MB-231 cells stably expressing hSulf2 and found decreased metabolic activity and reduced proliferation without change in cell viability (Fig. 2E) compared to control cells. Interestingly, treatment of MDA-MB-231 cells with rhSulf2 inhibited the growth of cells compared to controls (Fig. 2D) without affecting cell viability (Fig. 2F), similar to the effect of endogenously expressed hSulf2. This suggests that hSulf2 is probably interfering with growth factor stimulation of the cells, as previously demonstrated.

\section{HSulf2 inhibits growth factor-induced ERK activation}

It is speculated that sulfatases contribute to heparan sulfate remodeling on the tumor cell surface impairing growth factor signaling in the cells. It has been shown that hSulf1 in particular inhibits ERK1/2 phosphorylation through the FGF receptor. We wanted to determine the mechanism by which hSulf2 affects cell proliferation. MDA-MB-231 cells were treated with $250 \mathrm{nM}$ rhSulf2 for 24 hours prior to stimulation with FGF2 or HB-EGF. Cell lysates were collected before $(0 \mathrm{~min})$ or 15,30 , and 60 minutes following growth factor addition (Fig. 3). Treatment with rhSulf2 led to suppressed growth factorinduced phosphorylation of ERK1/2. Our data correlate with previous studies using hSulf1 that demonstrate inhibition of FGF signaling and growth both in vitro and in vivo in human cancer cells $[4,7]$.

\section{Stable expression of sulfatases in MDA-MB-231 cells inhibits in vivo tumor xenograft growth}

Expression of hSulf1 in human cancer cell xenograft in vivo inhibits tumor growth and progression [4,7]. No studies are currently available on the effect of hSulf2 on tumor xenograft in vivo. Therefore we tested two in vivo xenograft protocols to determine the effects of hSulf2 on tumor growth. As a positive control we used MDA-MB231 hSulf1 expressing cells. The two sulfatases were also co-expressed to test for a cooperative effect between hSulf1 and hSulf2. The stable transfectants were injected subcutaneously and monitored to compare sulfataseexpressing cells with empty vector control cells (Fig. 4). All groups formed tumors initially and there was no significant difference in average tumor volume at day 5 . Tumor xenografts composed of MDA-MB-231 cells stably co-expressing hSulf1 and hSulf2 $(\mathrm{S} 1+\mathrm{S} 2)$ demonstrated complete regression (Fig. $4, \mathrm{p}<0.02$ ), with no tumors remaining by day 35 after injection. Tumor xenograft groups expressing either hSulf1 or hSulf2 demonstrated partial regression with significant decreases in average tumor volume $(\mathrm{p}<0.03$ and $\mathrm{p}<0.02$ respectively). The fact that tumors co-expressing hSulf1 and hSulf2 formed tumors initially that later regressed suggests that a threshold of persistent sulfatase activity in the tumor microenvironment may be required for its anti-tumor activity. While MDA-MB231 xenograft growth rate and size varies amongst studies reported in the literature, our tumor xenografts were in range with those reported previously $[18,19]$. Tumor xenograft size variability and variable growth rate may be due to MDA-MB-231 cell line heterogeneity (as opposed to clonal expansion of a single cell population) as well as variation in strain of immunocompromised mouse host. $\mathrm{NCr}$ homozygous nude mice have intact innate immunity, which includes anti-tumor natural killer cell activity.

Control group tumors exhibited a significantly higher average mitotic index as demonstrated by Ki-67 staining (Fig. 4B, p $<0.001$ and 4 C). Control group tumors also displayed higher cellular density (white arrow in Fig. 5). Correlating with recent studies of sulfatase expression in myeloma cells [4], our experimental tumor xenograft groups expressing sulfatases show comparatively increased ECM deposition and collagen (black arrows in Fig. 5). Because of the possibility that tumor-derived sulfatases could impact both tumor and stromal cells, the vascular response in the tumors was evaluated by CD31 (PECAM) antibody staining of endothelial cells, followed by quantification of vessel area. There was no significant 

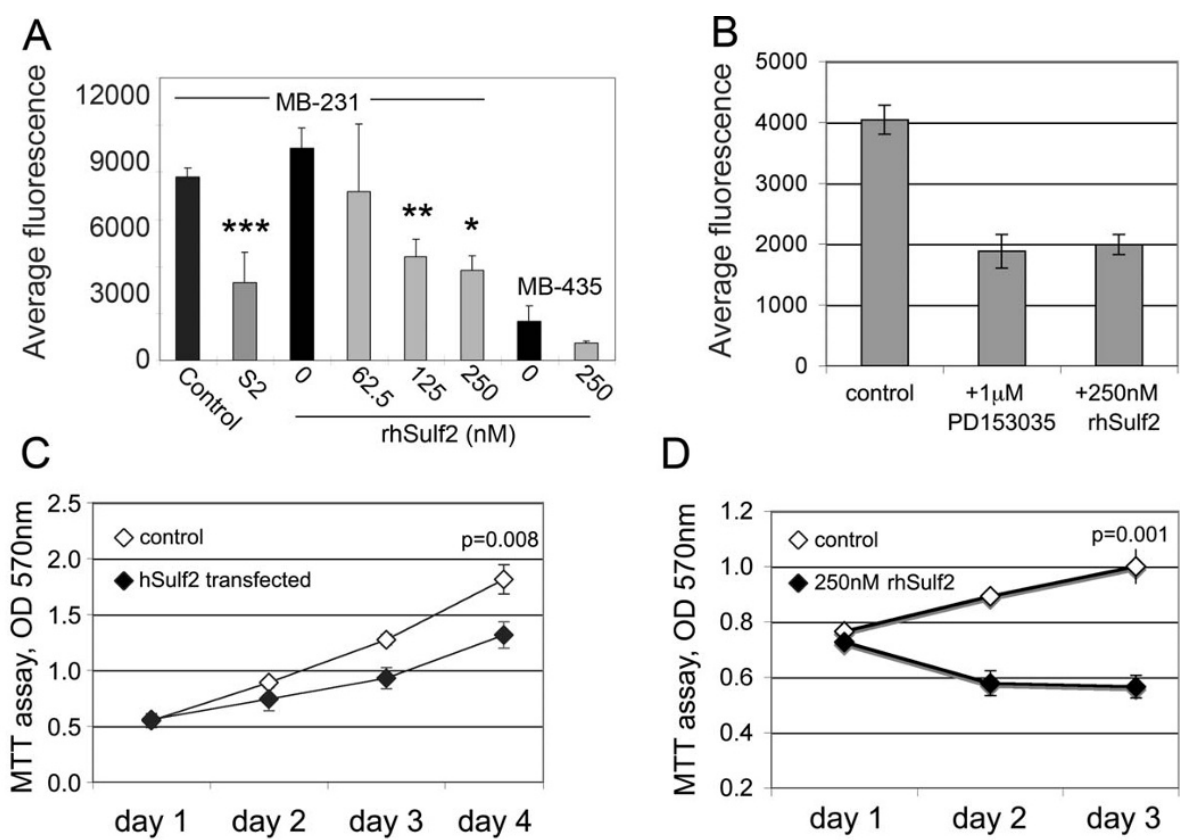

$\mathrm{E}$

$\mathrm{F}$
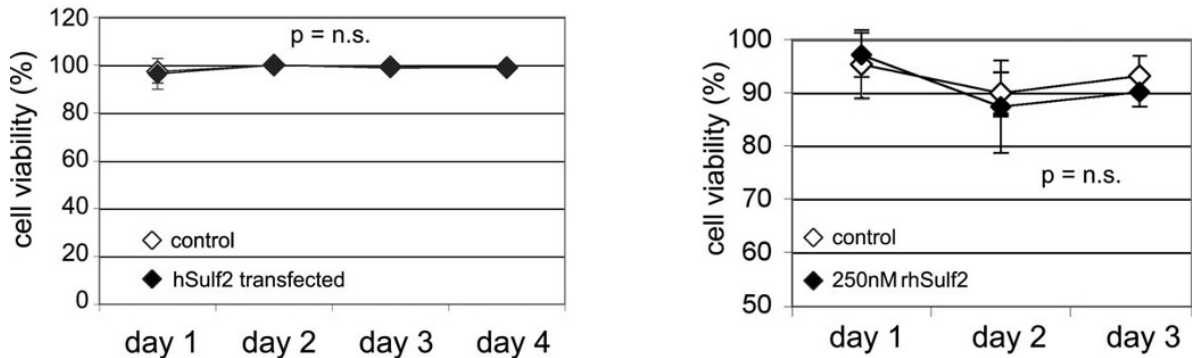

Figure 2 Sulfatase 2 decreases breast cancer cell invasion and proliferation. A) MDA-MB-231 (MB-231) and MDA-MB-435 S (MB-435) cell invasion was assayed with treatment of recombinant hSulf2 protein compared to buffer treatment (0). Stably hSulf2 transfected cells (S2) were also compared to control vector transfected cells for invasive ability ${ }^{*}=p<0.002 ;{ }^{* *}=p<0.001 ;{ }^{* * *}=p<0.04$. B) Invasion assays were performed with MDA-MB-231 cells in the presence of the EGFR kinase inhibitor PD153035 or rhSulf2. C) For the proliferation assay, cells were plated and collected over 4 days to test mitochondrial reductase metabolic activity using the MTT assay. Decreased MTT conversion in hSulf2 transfected cells corresponded to non-proliferating cells during this period. D) Recombinant human Sulfatase 2 protein (rhSulf2) was added to MDA-MB-231 cells at $250 \mathrm{nM}$, and cells assayed over 3 days using the MTT assay. E) Cell viability is expressed as percent of live cells and corresponds to data represented in panel C. F) Corresponding cell viability data for panel D; n.s. = not significant.
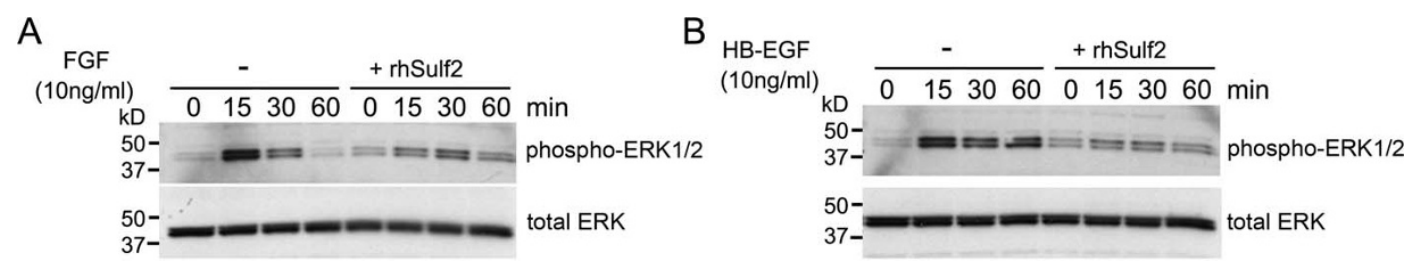

Figure 3 Recombinant human Sulfatase 2 inhibits growth factor-induced ERK activation. MDA-MB-231 cells were treated with $250 \mathrm{nM}$ rhSulf2 for $24 \mathrm{~h}$ prior to FGF (A) or HB-EGF (B) stimulation. Cell lysates were collected before growth factor addition ( 0 min), or 15, 30, and 60 minutes after addition for analysis of phospho-ERK1/2 and total ERK. The two phosphorylated p44 and p42 species of ERK are detected using phospho-ERK1/2 antibodies. 

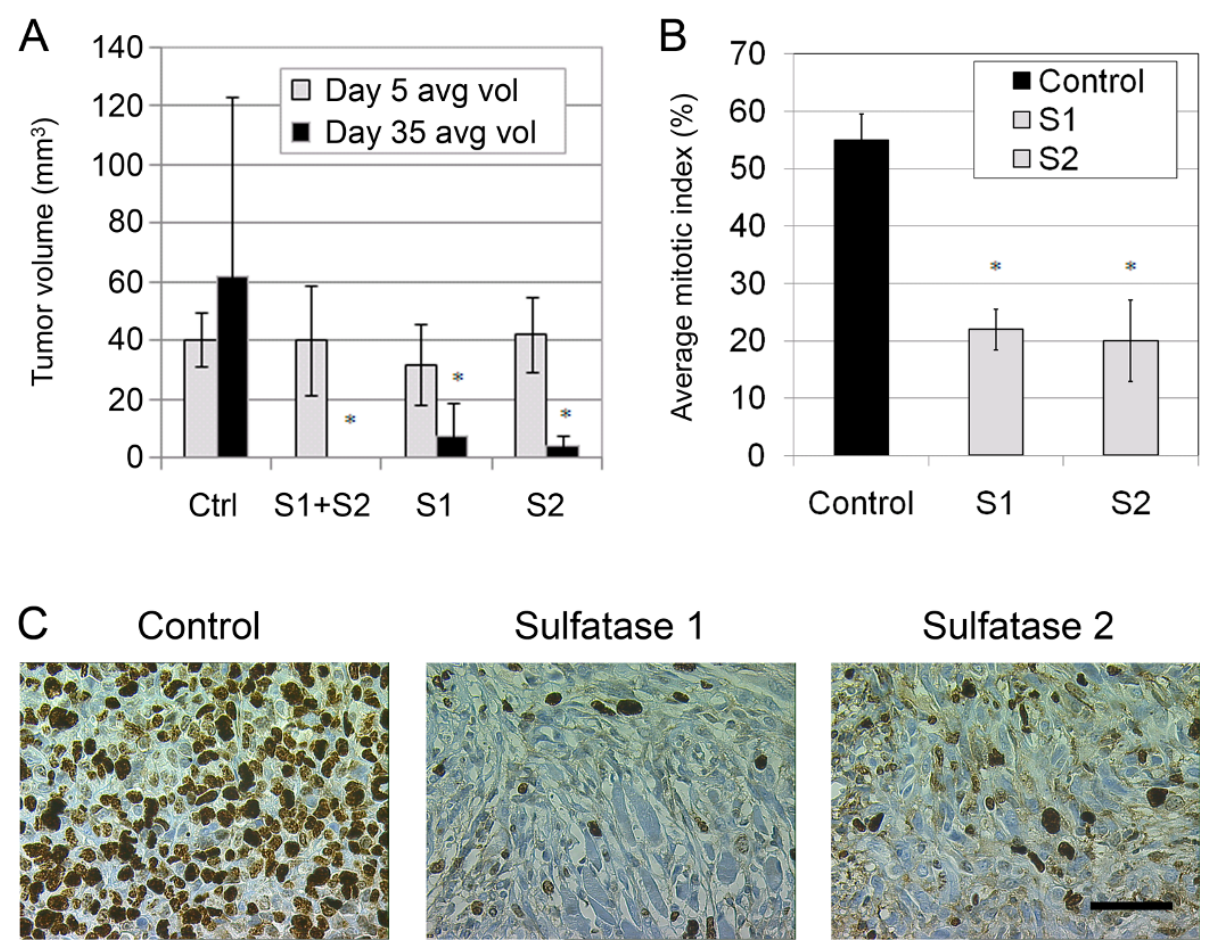

Figure 4 Stable expression of sulfatases in MDA-MB-231 cells inhibits in vivo tumor xenograft growth. A) Average tumor volume at day 35 study endpoint for control vector transfected MDA-MB-231 cells compared with hSulf1 and hSulf2 transfected (S1+S2), hSulf1 (S1), and hSulf2 (S2) transfected cells. The average value and standard deviation are based on caliper measurements and calculated tumor volumes from the eight mice in each group * $p<0.03$. B) Average mitotic index at day 35 study endpoint for hSulf1 (S1) and hSulf2 (S2) transfected tumors in comparison to control as determined by Ki-67 staining ${ }^{*} p<0.001$. C) Representative fields used for quantification of mitotic index by Ki- 67 staining. The scale bar represents $50 \mu \mathrm{m}$.

difference in average microvascular density among tumors in the control, hSulf1 or hSulf2 transfected groups (data not shown). This finding correlates with the observation in myeloma tumor xenografts that inhibition of tumor xenograft formation was not dependent on changes in microvessel density [4]. Although in vitro cell viability was not regulated by sulfatases, the in vivo phenotype shows tumor regression following the establishment of a small tumor, particularly with the combined expression of hSulf1 and hSulf2. Thus, hSulf2 expression in tumor cells leads to a phenotype consistent with cell intrinsic and localized effect to suppress growth of tumor cells. Dai et al. postulate that restricted sulfatase activity within the tumor microenvironment in vivo is due to heparan sulfate remodeling on the tumor cell surface rather than the surrounding ECM [4]. Experimental support for this model was the assembly of an FGF2 tertiary signaling complex in the tumor stroma but not on the surface of tumor cells [4]. This is further supported by the fact that a restricted local effect of Sulf1 has been shown by Ai, et al. who demonstrated that quail Sulf1 only alters the heparan sulfate on the cell expressing the enzyme as opposed to adjacent cells that lack Sulf1 expression [20].
Exogenous short term intratumoral administration of rhSulf2 is not sufficient for inhibition of tumor xenograft growth

As a second method to test sulfatase activity in tumor growth, we formed xenografts with MDA-MB-231 cells (non-transfected), and treated growing tumors by intratumoral injection of rhSulf2 or buffer vehicle. RhSulf2 injections were started 2 days after cell injection, and continued for 7 consecutive days. There was no significant difference in average tumor volume between the rhSulf2 treated mice or the buffer vehicle treated mice at day 9 after tumor cell injection (Fig. 6A). The differences observed with this protocol versus the stable transfectants may be due to lack of persistent, tumorderived sulfatase expression. As noted above, the source of the sulfatase and its ability to mediate functional enzymatic activity seems to require activity near tumor cell surfaces. This may explain why transfected tumor cells exhibited dramatic inhibition of tumor xenograft growth while it was not possible to mimic these results with exogenously administered rhSulf2 into the general tumor environment. In addition, the variability between individual tumor growth patterns in this experiment could also account for lack of significant decrease in 


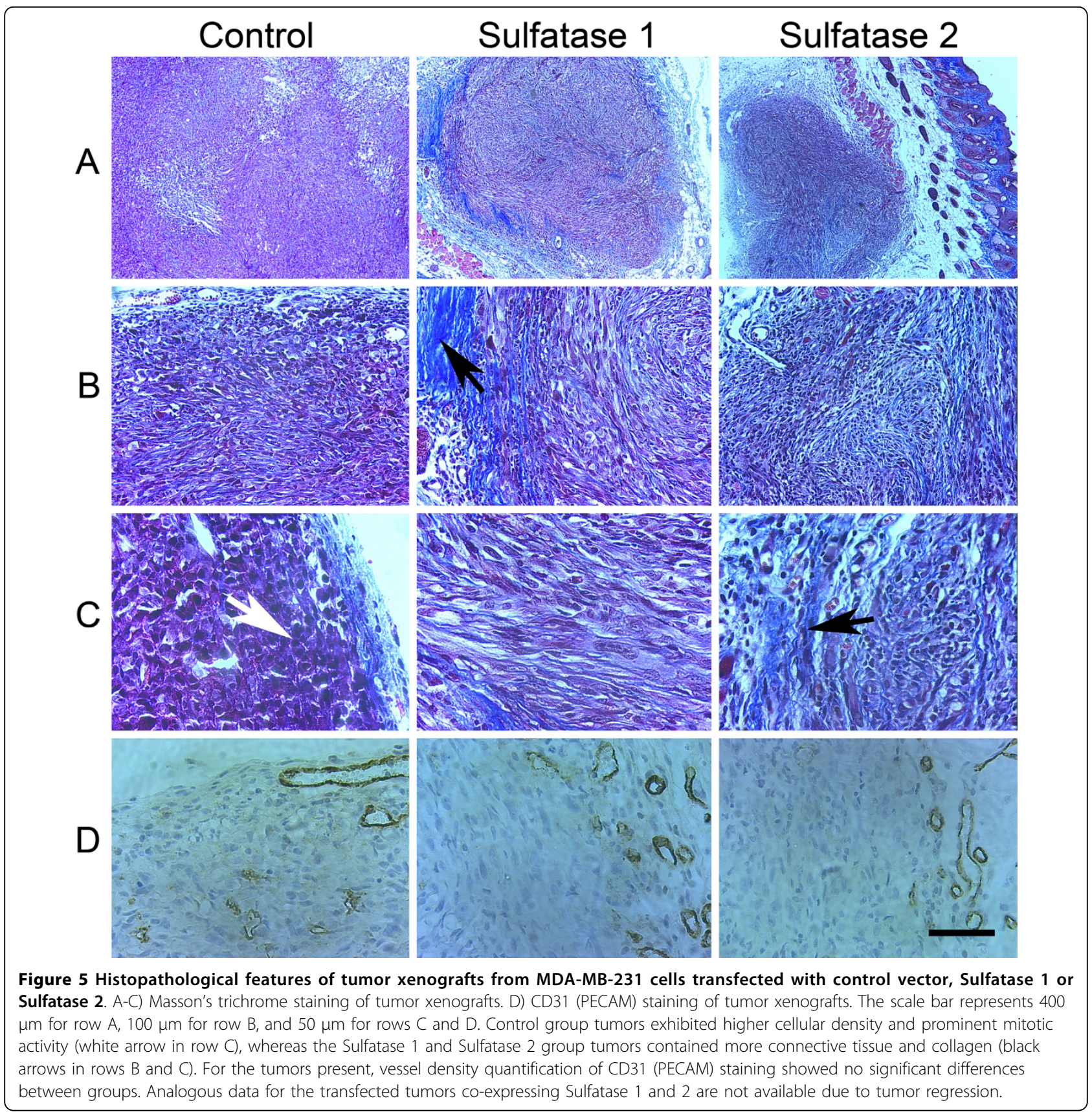

tumor volumes after a short treatment period. Average tumor volume was additionally obtained by threedimensional (3D) reconstruction of the tumor by highresolution ultrasound scanning. As expected, control mice not injected with cells or buffer-matrigel suspension did not develop spontaneous tumors. The control mice injected with buffer-matrigel suspension initially developed palpable nodules at the injection site due to the matrigel suspension that spontaneously regressed within 9 days (Fig. 6A). For simplicity, the control data point is based on the eight mice included in both control branches. These studies validate the use of ultrasound as a highly quantitative method to monitor 3D growth of tumors over time. This method may be particularly useful for the tracking of tumors growing internally on or within solid organs.

\section{Discussion and Conclusions}

The results presented in this study suggest a potential role for hSulf2 in inhibiting cancer growth and metastases. We have demonstrated that cell invasion through an ECM-enriched matrigel is strongly inhibited when 


\section{A}

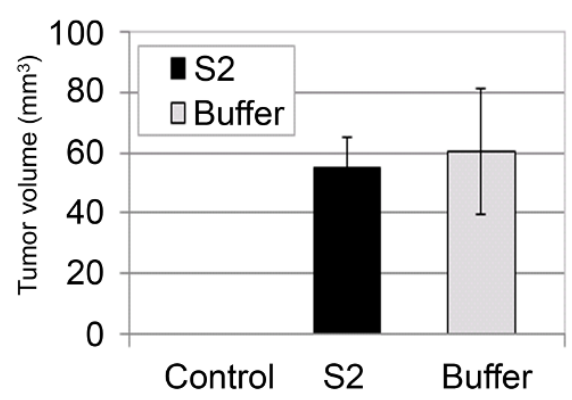

C

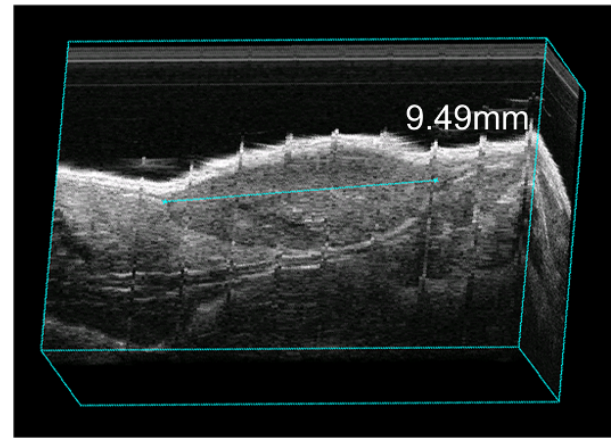

B

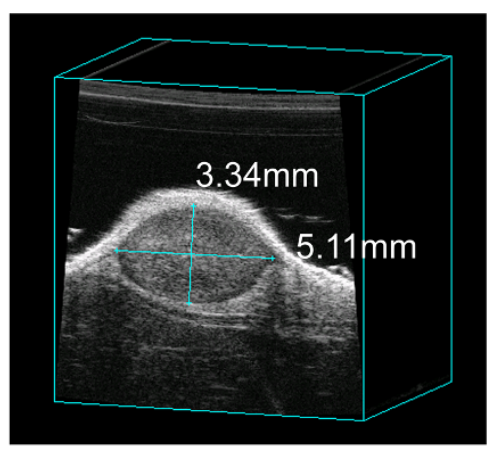

D

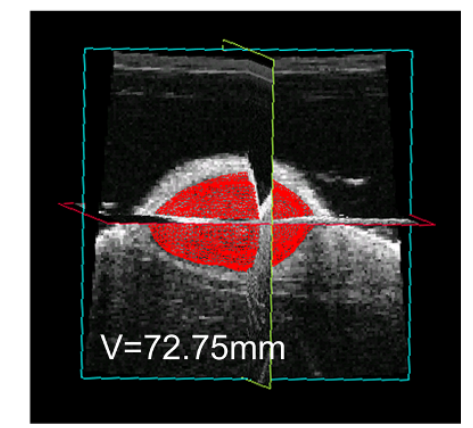

Figure 6 Exogenous treatment with short course therapy of purified hSulf-2 insufficient for tumor xenograft inhibition. Athymic nude mice were randomized into two experimental groups ( $n=16$ each) and injected with MDA-MB-231 cells. On day 2 after injection, intratumoral injections were started with rhSulf2 or equivalent volume of vehicle. No significant difference was noted in average tumor xenograft volume after short course intratumoral injections of purified rhSulf2. Average volume as estimated by high resolution 3D ultrasound scanning of tumors at day 9 (A). Two methods for estimation of tumor size are shown in panels B-D. In the first method, width and height measurements (B) in combination with length (C) can be used in ellipsoid volume calculations as described above. Alternatively, tumor tracings in multiple frames (C) can be used to recreate a 3-dimensional tumor reconstruction and estimated volume (E). Given the irregular nature of these tumors, this second method was employed for capturing aberrancies from true ellipsoid shape. In this case, ultrasound-estimated volume is $\sim 73 \mathrm{~mm}^{3}$ compared to an equation-calculated volume of $\sim 85 \mathrm{~mm}^{3}$. This smaller volume is not surprising considering that caliper measurements include the overlying subcutaneous tissue whereas intra-ultrasound caliper measurements measure only the tumor itself.

the cells are expressing hSulf2 or are in the presence of rhSulf2, and this effect is dose-dependent. The inhibitory effect of hSulf2 on MDA-MD-231 cells is comparable to the one observed with the classical EGFR-dependent kinase inhibitor PD153035. HSulf2 inhibits cell growth and proliferation of cells, and it does so by interfering with growth factor signaling.

Until recently, investigations into the action of hSulf1 and hSulf2 focused on growth factor modulation and angiogenesis. In the non-sulfatase expressing breast cancer cell line MDA-MB-468, forced expression of hSulf1 led to inhibition of angiogenesis and tumorigenesis in xenografts [8]. While there is consensus regarding the action of hSulf1 and hSulf2 in the desulfation of heparan sulfate, there is controversy over whether this effect is pro-angiogenic $[6,21]$ or anti-angiogenic $[4,6,8,10,21]$. Specifically, studies in carcinoma cell lines producing hSulf2 demonstrate that MCF-7-derived hSulf2 decreases heparin sequestration of VEGF, FGF1 and selected chemokines to promote growth factor activity by prevention of binding and dissociation of complexes that are already bound [21]. Similarly, Sulf2 promotes angiogenesis in the chick chorioallantoic membrane assay [6]. In contrast, studies of other breast, pancreatic, renal, and hepatocellular carcinoma cell lines in which Sulf1 is down-regulated, suggest that endosulfatase activity inhibits angiogenesis. One potential explanation for this is that sulfatases may display additional effects beyond modulation of angiogenesis in specific contexts.

Interestingly, hSulf2 has recently been identified as a novel transcriptional target of the tumor suppressor gene p53 indicating that it may be a p53 downstream effector molecule [8]. This finding is particularly significant in the context of mutant p53 status in MDA-MB231 and MDA-MB-468 cells and wild-type p53 status in MCF-7 breast cancer cell lines [22,23]. Knockdown of mutant p53 by RNA interference causes massive apoptosis in mutant p53 breast cancer cell lines but not in wild-type p53 breast cancer cell lines, indicating that mutant p53 may confer oncogenic potential as well as loss of tumor suppressor activity [23]. Knock-down of Sulf2 in MDA-MB-231 cells confers enhanced survival 
characterized by increased proliferation and anchorageindependent growth [11]. Our data are consistent with this work, because expression of hSulf2 had the opposite phenotype in decreasing tumor cell growth. Our findings extend the in vitro work to provide the first description of in vivo breast cancer xenograft growth inhibition by sulfatases.

Ai et al. [24] demonstrated that avian endosulfatases, Sulf1 and Sulf2, associate with the cell membrane and are enzymatically active on the cell surface to desulfate HSPG. Desulfation of HSPG by endosulfatases has been shown to impair growth factor signaling through FGF and EGF receptors [4,7]. In this study we have demonstrated that rhSulf2 decreases ERK1/2 phosphorylation as previously found for hSulf1 and hSulf2. Therefore, our data support decreased growth factor signaling as the mechanism of inhibition of tumor growth. Combinatorial therapy using conventional chemotherapy and hSulf2 could provide an alternative and more effective option, although further study is needed to determine whether hSulf2 could potentially be an effective exogenous therapy for specific subtypes of breast adenocarcinoma.

While data from sulfatase-transfected tumors are promising, a detailed in vivo study is required to reproduce these results with exogenous administration of rhSulf2. Although short term intratumoral administration of rhSulf2 was not efficient in inhibiting tumor growth, our data suggest that constant production of hSulf2 is required for a theraupeutic effect. In addition, secretion of hSulf2 in stable transfectants from early stages of xenograft growth may have modified the tumor cells or microenvironment. Although we performed a pilot study to administer rhSulf 2 by intravenous tail vein injection, this approach was limited by the high osmolarity of buffer vehicle in a tail vein injection distal enough to allow for adequate proximal intravenous access for subsequent daily delivery (data not shown). In addition, the concentrations of purified proteins were not sufficiently high to allow for injection of small enough volumes. While this could potentially be circumvented by jugular venous cannulation for central line access, the concentration of protein accumulated at the tumor site is predicted to be much lower than compared to intratumoral injection. Development of strategies for therapeutic exogenous administration of sulfatases requires in depth pharmacokinetic analysis for determination of volume of distribution, rate of metabolism, and dose-response curves in different contexts. Given the complexity of cellular events during malignant transformation and tumor progression in addition to the context-dependent nature of sulfatase activity, it is not surprising that the delivery method is critical to tumor response. Potential complicating factors to be considered in the future include active concentrations in the tumor microenvironment, the timing and duration of sulfatase administration, and a requirement for tumor cell expression for intrinsic growth effect. However, our studies provide proof of principle that sulfatase within a human breast cancer xenograft can lead to tumor suppression and indeed regression of established tumors.

\section{Abbreviations}

HSPG: heparin/heparan sulfate proteoglycan; FGF: fibroblast growth factor; EGF: epidermal growth factor; VEGF: vascular endothelial growth factor; Sulf2: Sulfatase 2; hSulf2: human Sulfatase 2; rhSulf2: recombinant human Sulfatase 2; Sulf1: Sulfatase 1; hSulf1: human Sulfatase 1; MB 231: MDA-MB-231 breast cancer cell line; ERK: extracellular signal-related kinases; ECM: extracellular matrix; HCC: hepatocellular carcinoma; HGF: hepatocyte growth factor; RT$P C R$ : reverse transcription polymerase chain reaction; HB-EGF: heparinbinding epidermal growth factor-like growth factor; EGFR: epidermal growth factor receptor; MEM: minimal essential media; FBS: fetal bovine serum; MTT: 3-(4,5-Dimethylthiazol-2-yl)-2,5-diphenyltetrazolium bromide; DMSO: dimethyl sulfoxide; OD: optical density; PBS: phosphate buffered saline; 4-MUS: 4methylumbelliferyl sulfate; 3D: three-dimensional; RNA: ribonucleic acid.

\section{Acknowledgements}

We gratefully acknowledge the support of the Maine Medical Center Research Institute Histopathology Core (Dr. Volkhard Lindner and Kathleen Carrier).

Financial Support: Shire, The Histopathology Core Facility at Maine Medical Center Research Institute is supported by P20RR181789 (PI: D. Wojchowski) from the National Center for Research Resources at NIH.

\section{Author details}

${ }^{1}$ Maine Medical Center Research Institute, 81 Research Drive, Scarborough, ME 04074, USA. ${ }^{2}$ Shire Human Genetic Therapies Inc., 700 Main Street, Cambridge, MA 02139, USA.

\section{Authors' contributions}

PGVM conceived the idea of this study. PGVM, Al, LC, AN, AR, MFC, MWH and $A G Y$ designed and/or performed the in vitro experiments. SMP, KT, and $\mathrm{LL}$ designed and performed the in vivo experiments. SMP and LL drafted the initial manuscript, prepared the figures, an edited subsequent revisions. MJ performed the histopathology analysis. PGVM critically revised the manuscript. All authors read and approved the final manuscript.

\section{Competing interests}

Financial disclosure: Project funding was provided by Shire Pharmaceuticals. Al, LC, AR, AN, AG-Y, MWH, MFC, and PGVM are current employees of Shire Human Genetic Therapies Inc.

Received: 4 January 2010 Accepted: 13 August 2010

Published: 13 August 2010

\section{References}

1. Goldman M, O'Hair K: Women's health, breast health: a review of the gynecologic effects of breast cancer. Obstet Gynecol Surv 2009, 64(7):469-480, quiz 499.

2. Frese MA, Milz F, Dick M, Lamanna WC, Dierks T: Characterization of the human sulfatase Sulf1 and its high affinity heparin/heparan sulfate interaction domain. J Biol Chem 2009, 284(41):28033-28044.

3. Liu D, Shriver Z, Venkataraman G, El Shabrawi Y, Sasisekharan R: Tumor cell surface heparan sulfate as cryptic promoters or inhibitors of tumor growth and metastasis. Proc Natl Acad Sci USA 2002, 99(2):568-573.

4. Dai Y, Yang Y, MacLeod V, Yue X, Rapraeger AC, Shriver Z, Venkataraman G, Sasisekharan R, Sanderson RD: HSulf-1 and HSulf-2 are potent inhibitors of myeloma tumor growth in vivo. J Biol Chem 2005, 280(48):40066-40073.

5. Chau BN, Diaz RL, Saunders MA, Cheng C, Chang AN, Warrener P, Bradshaw J, Linsley PS, Cleary MA: Identification of SULF2 as a novel transcriptional target of $\mathrm{p} 53$ by use of integrated genomic analyses. Cancer Res 2009, 69(4):1368-1374. 
6. Morimoto-Tomita M, Uchimura K, Bistrup A, Lum DH, Egeblad M, Boudreau N, Werb Z, Rosen SD: Sulf-2, a proangiogenic heparan sulfate endosulfatase, is upregulated in breast cancer. Neoplasia 2005, 7(11):1001-1010.

7. Lai J, Chien J, Staub J, Avula R, Greene EL, Matthews TA, Smith DI, Kaufmann SH, Roberts LR, Shridhar V: Loss of HSulf-1 up-regulates heparin-binding growth factor signaling in cancer. J Biol Chem 2003, 278(25):23107-23117.

8. Narita K, Staub J, Chien J, Meyer K, Bauer M, Friedl A, Ramakrishnan S, Shridhar V: HSulf-1 inhibits angiogenesis and tumorigenesis in vivo. Cancer Res 2006, 66(12):6025-6032.

9. Holst CR, Bou-Reslan H, Gore BB, Wong K, Grant D, Chalasani S, Carano RA, Frantz GD, Tessier-Lavigne M, Bolon B, et al: Secreted sulfatases Sulf1 and Sulf2 have overlapping yet essential roles in mouse neonatal survival. PLoS One 2007, 2(6):e575.

10. Lai JP, Chien JR, Moser DR, Staub JK, Aderca I, Montoya DP, Matthews TA, Nagorney DM, Cunningham JM, Smith DI, et al: hSulf1 Sulfatase promotes apoptosis of hepatocellular cancer cells by decreasing heparin-binding growth factor signaling. Gastroenterology 2004, 126(1):231-248.

11. Hampton OA, Den Hollander P, Miller CA, Delgado DA, Li J, Coarfa C, Harris RA, Richards S, Scherer SE, Muzny DM, et al: A sequence-level map of chromosomal breakpoints in the MCF-7 breast cancer cell line yields insights into the evolution of a cancer genome. Genome Res 2009, 19(2):167-177.

12. Tomayko MM, Reynolds CP: Determination of subcutaneous tumor size in athymic (nude) mice. Cancer Chemother Pharmacol 1989, 24(3):148-154.

13. ImageJ. [http://rsb.info.nih.gov/ij].

14. Miki $Y$, Suzuki T, Sasano H: Intracrinology of sex steroids in ductal carcinoma in situ (DCIS) of human breast: comparison to invasive ductal carcinoma (IDC) and non-neoplastic breast. J Steroid Biochem Mol Biol 2009, 114(1-2):68-71.

15. Banerjee S, Dowsett M, Ashworth A, Martin LA: Mechanisms of disease: angiogenesis and the management of breast cancer. Nat Clin Pract Oncol 2007, 4(9):536-550.

16. Morimoto-Tomita M, Uchimura K, Werb Z, Hemmerich S, Rosen SD: Cloning and characterization of two extracellular heparin-degrading endosulfatases in mice and humans. J Biol Chem 2002, 277(51):49175-49185.

17. Gangarosa LM, Sizemore N, Graves-Deal R, Oldham SM, Der CJ, Coffey RJ: A raf-independent epidermal growth factor receptor autocrine loop is necessary for Ras transformation of rat intestinal epithelial cells. J Biol Chem 1997, 272(30):18926-18931.

18. Fraker LD, Halter SA, Forbes JT: Growth inhibition by retinol of a human breast carcinoma cell line in vitro and in athymic mice. Cancer Res 1984, 44(12 Pt 1):5757-5763

19. Wang J, Ou ZL, Hou YF, Luo JM, Shen ZZ, Ding J, Shao ZM: Enhanced expression of Duffy antigen receptor for chemokines by breast cancer cells attenuates growth and metastasis potential. Oncogene 2006, 25(54):7201-7211.

20. Ai X, Do AT, Lozynska O, Kusche-Gullberg M, Lindahl U, Emerson CP Jr: QSulf1 remodels the 6-O sulfation states of cell surface heparan sulfate proteoglycans to promote Wnt signaling. J Cell Biol 2003, 162(2):341-351.

21. Uchimura K, Morimoto-Tomita M, Bistrup A, Li J, Lyon M, Gallagher J, Werb Z, Rosen SD: HSulf-2, an extracellular endoglucosamine-6-sulfatase, selectively mobilizes heparin-bound growth factors and chemokines: effects on VEGF, FGF-1, and SDF-1. BMC Biochem 2006, 7:2.

22. Hui L, Zheng $Y$, Yan $Y$, Bargonetti J, Foster DA: Mutant p53 in MDA-MB231 breast cancer cells is stabilized by elevated phospholipase $D$ activity and contributes to survival signals generated by phospholipase D. Oncogene 2006, 25(55):7305-7310.

23. Lim LY, Vidnovic N, Ellisen LW, Leong CO: Mutant p53 mediates survival of breast cancer cells. Br J Cancer 2009, 101:1606-1612.

24. Ai X, Do AT, Kusche-Gullberg M, Lindahl U, Lu K, Emerson CP Jr: Substrate specificity and domain functions of extracellular heparan sulfate 6-Oendosulfatases, QSulf1 and QSulf2. J Biol Chem 2006, 281(8):4969-4976.

\section{Pre-publication history}

The pre-publication history for this paper can be accessed here: http://www.biomedcentral.com/1471-2407/10/427/prepub

doi:10.1186/1471-2407-10-427

Cite this article as: Peterson et al:: Human Sulfatase 2 inhibits in vivo tumor growth of MDA-MB-231 human breast cancer xenografts. BMC Cancer 2010 10:427.

\section{Submit your next manuscript to BioMed Central and take full advantage of:}

- Convenient online submission

- Thorough peer review

- No space constraints or color figure charges

- Immediate publication on acceptance

- Inclusion in PubMed, CAS, Scopus and Google Scholar

- Research which is freely available for redistribution

Submit your manuscript at www.biomedcentral.com/submit
C) Biomed Central 